\title{
Three new species of Willowsia (Collembola: Entomobryidae) from Guizhou Province, China
}

\author{
Yan $\mathrm{CHANG}^{1}$ \& Yitong $\mathrm{MA}^{2, *}$ \\ ${ }^{1,2}$ School of Life Sciences, Nantong University, Nantong 226000, P.R. China. \\ *Corresponding author: mayitong@ntu.edu.cn \\ ${ }^{1}$ Email: cuckoe@ntu.edu.cn \\ ${ }^{1}$ urn:lsid:zoobank.org:author:E68EE28F-3773-4123-BBEC-320461569E2B \\ ${ }^{2}$ urn:Isid:zoobank.org:author:14F92F90-863A-46EB-B056-41EB3FA341A0
}

\begin{abstract}
Three new species of Willowsia collected from Guizhou Province, China are described here: $W$. sexachaeta sp. nov., W. christianseni sp. nov., and W. tanae sp. nov. They have spinulate scales on the body. Colour pattern and dorsal chaetotaxy are the main diagnostic characters for these species. A table summarizing the main differences between all Chinese Willowsia species is given.
\end{abstract}

Keywords. Entomobryinae, taxonomy, chaetotaxy, scales, new species.

Chang Y. \& Ma Y. 2018. Three new species of Willowsia (Collembola: Entomobryidae) from Guizhou Province, China. European Journal of Taxonomy xxx: 1-18. https://doi.org/10.5852/ejt.2018.470

\section{Introduction}

The genus Willowsia Shoebotham, 1917 belongs to the family Entomobryidae and has the following main characters: eyes $8+8$, dens without spines or scales, mucro bidentate with a basal spine, scales present on body. Scale morphology is diversified in Willowsia. Zhang et al. (2011) divided scales into four types based on surface sculpture: spinulate type, short rib type, long basal rib type and uninterrupted type.

Thirty-eight species have been described worldwide, ranging from the Arctic region to tropical areas. Among them, 26 species are from Asia and 17 from China. In this study, three new species from Guizhou Province, China are described: $W$. sexachaeta sp. nov., $W$. christianseni sp. nov. and $W$. tanae sp. nov. Similar to other Willowsia species from Guizhou Province, these new species also have scales of the spinulate type. Table 1 summarizes the main differences between all Chinese species of Willowsia.

\section{Material and methods}

After clearing in lactic acid, specimens were mounted under a coverslip in Marc André II solution, and were studied with a Leica DM2500 microscope. Images were taken with a Hitachi SN3400 scanning electron microscope and a mounted Leica DFC300 FX digital camera, and enhanced with Photoshop CS2 (Adobe Inc.). The number of macrochaetae is given by half-tergite in the descriptions. The nomenclature 


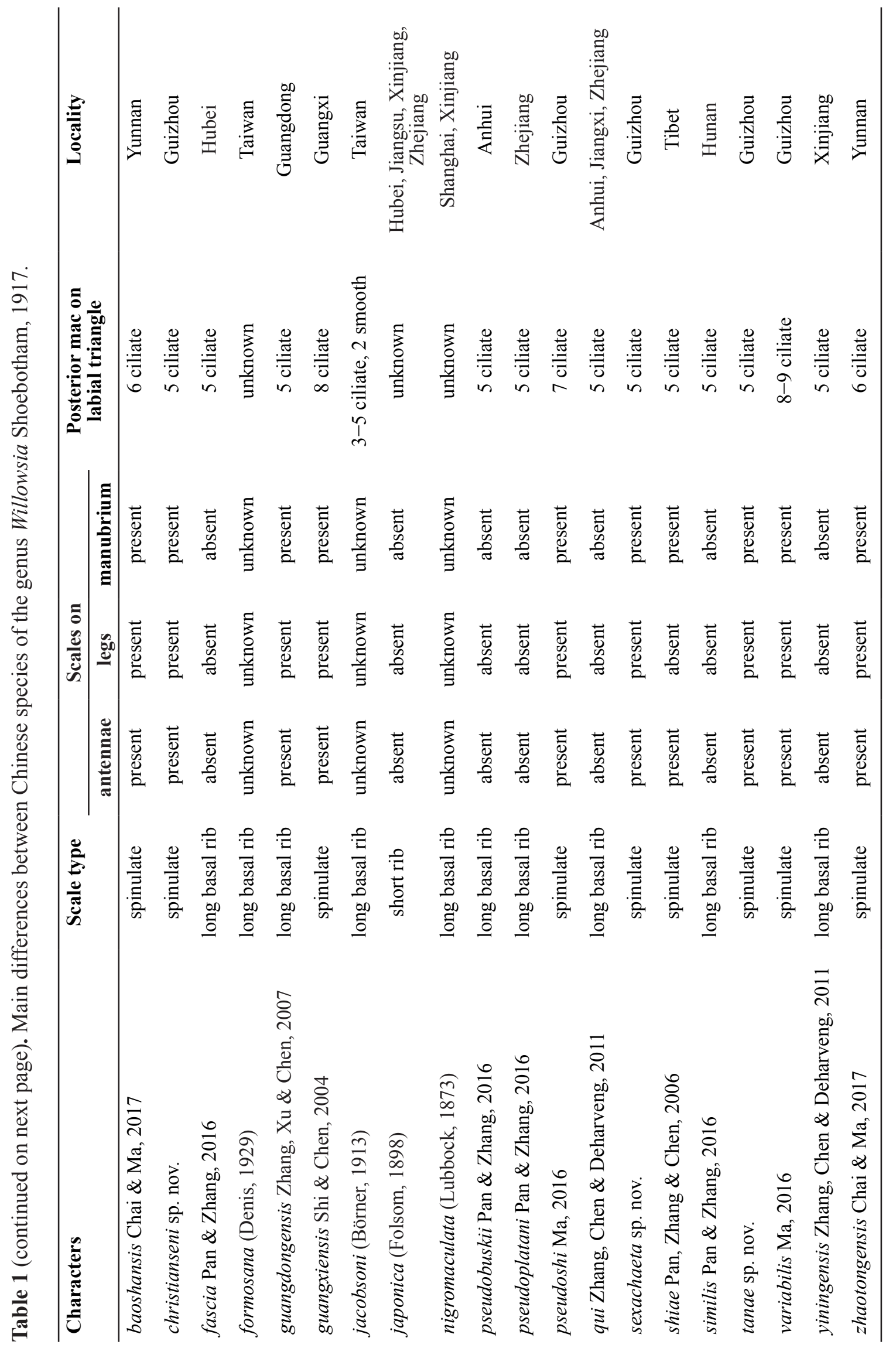




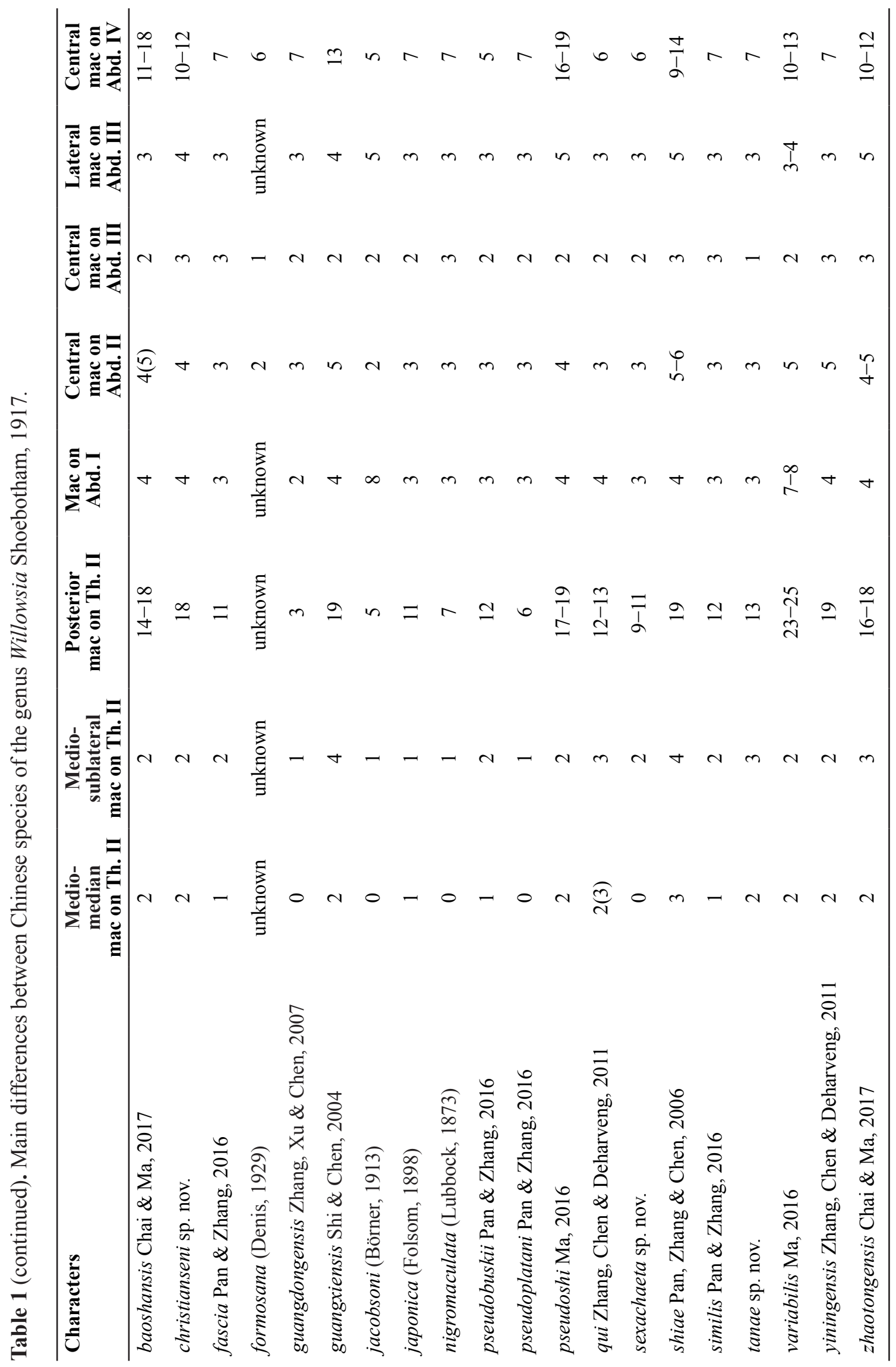


of the dorsal chaetotaxy of the head and interocular chaetae is described following Jordana \& Baquero (2005) and Mari-Mutt (1986). Labial chaetae are designated following Gisin (1967). Tergal chaetae of the body are designated using the system of Szeptycki (1979).

\section{Abbreviations}

Abd. $=$ abdominal segment

Ant. = antennal segment

Mac $=$ macrochaeta $(\mathrm{e})$

$\mathrm{ms}=\mathrm{S}$-microchaeta(e) (microsensillum, $-\mathrm{a})$

sens $=$ ordinary S-chaeta(e)

Th. $=$ thoracic segment

\section{Institutional abbreviations}

NTU $=$ Nantong University, Nantong, China

\section{Results}

Class Collembola Lubbock, 1873

Order Entomobryomorpha Börner, 1913

Family Entomobryidae Tömösvary, 1882

Subfamily Entomobryinae Schäffer, 1896

Genus Willowsia Shoebotham, 1917

\section{Diagnosis}

Moderate size, usually 1-2 mm; eyes $8+8$; antennae four segmented; mucro bidentate with a basal spine; dens without spines or scales; body with various types of scales in different species.

Willowsia sexachaeta sp. nov.

urn:1sid:zoobank.org:act:972AD9E1-C147-43A0-ADA4-184AEB45550B

Figs $1-4$, Tables $1-2$

\section{Diagnosis}

A longitudinal stripe present from eyepatch to Th. III along lateral side; Abd. I with 3 mac; Abd. II with 3 central mac; Abd. III with 2 central and 3 lateral mac; scales spinulate type and present on Ant. I-II, dorsal side of head and terga, coxa to tibiotarsus, anterior face of ventral tube and ventral side of manubrium.

\section{Etymology}

Named after the characteristic six (Latin word "sex-") macrochaetae present on the central part of Abd. IV.

\section{Type material}

\section{Holotype}

CHINA: + , on slide, Louna Village, Dingxiao Town, Xingyi City, Guizhou Province, $25^{\circ} 09^{\prime} 33^{\prime \prime}$ N, $105^{\circ} 02^{\prime} 35^{\prime \prime}$ E, $1300 \mathrm{~m}$ a.s.1., in rotten stem of maize and litter, 21 Apr. 2014, Shangze Wu leg. (collection number 1132, NTU). 
Table 2. Comparison of $W$. sexachaeta sp. nov., W. baoshanensis Chai \& Ma, 2017 and W. japonica Folsom 1898.

\begin{tabular}{lccc}
\hline Characters & W. sexachaeta sp. nov. & W. baoshanensis & W. japonica \\
\hline Scale type & spinulate & spinulate & short rib \\
Scales on antennae, & present & present & absent \\
legs and manubrium & absent & present & present \\
Mac m2 on Th. II & present & present & absent \\
Mac m4i on Th. II & absent & absent & present \\
Mac a2 \& a3 on Th. III & 3 & 4 & 3 \\
Mac on Abd. I & 3 & $4(5)$ & 3 \\
Central mac on Abd. II & 6 & $11-18$ & 7 \\
Central mac on Abd. IV & $1.4-1.7$ & $1.5-1.7$ & $2.2-2.9$ \\
Uncrenulated dens/ mucro & &
\end{tabular}

\section{Paratypes}

CHINA: 9 우, on slides, same data as holotype.

\section{Description}

Measurments. Body length up to $1.6 \mathrm{~mm}$.

CoLour. Ground colour pale yellow. Antennae with scattered blue pigment, especially distal part of each segment. Eyepatches dark blue. Longitudinal stripe present from eyepatch to Th. III along lateral side (Figs 1A-1B).

HEAD. Antenna $0.4-0.6 \times$ as long as body. Antennal segments ratio as I: II: III: IV $=1.0: 1.7-2.2$ : 1.7-2.3: 2.8-3.5. Distal part of Ant. IV with many sensory chaetae, normal ciliate chaetae and a bilobed apical bulb (Fig. 2A). Dorsal cephalic chaetotaxy with 5-7 antennal (An), 4 median (M) and 8 sutural (S) mac. Interocular chaetae as p, s, t, all finely ciliate (Fig. 2B). Eyes 8+8, G and H smaller (Fig. 2B).

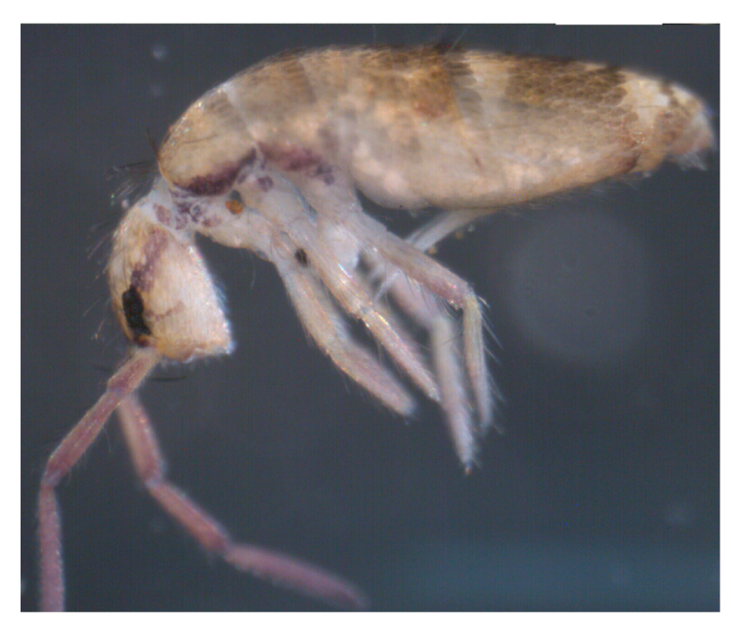

A

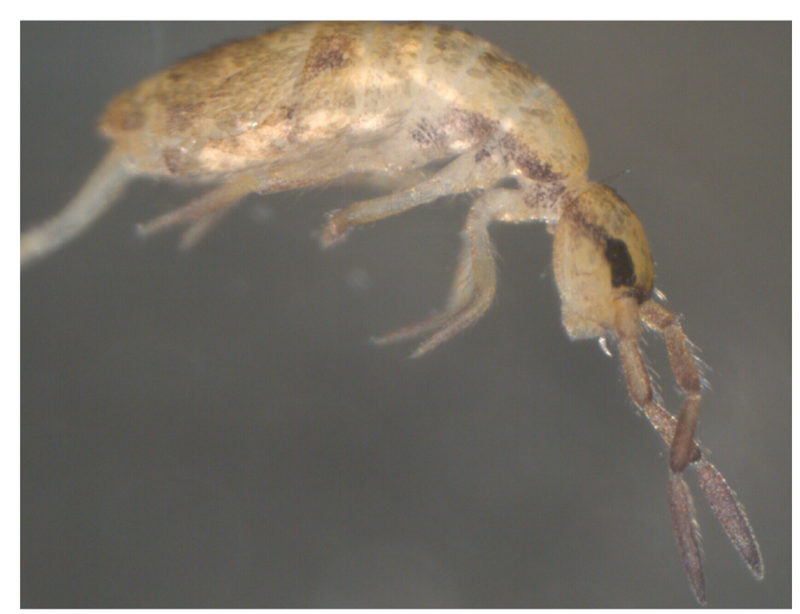

$\mathrm{B}$

Fig. 1. Willowsia sexachaeta sp. nov. A-B. Habitus. Scale bars: $500 \mu \mathrm{m}$. 

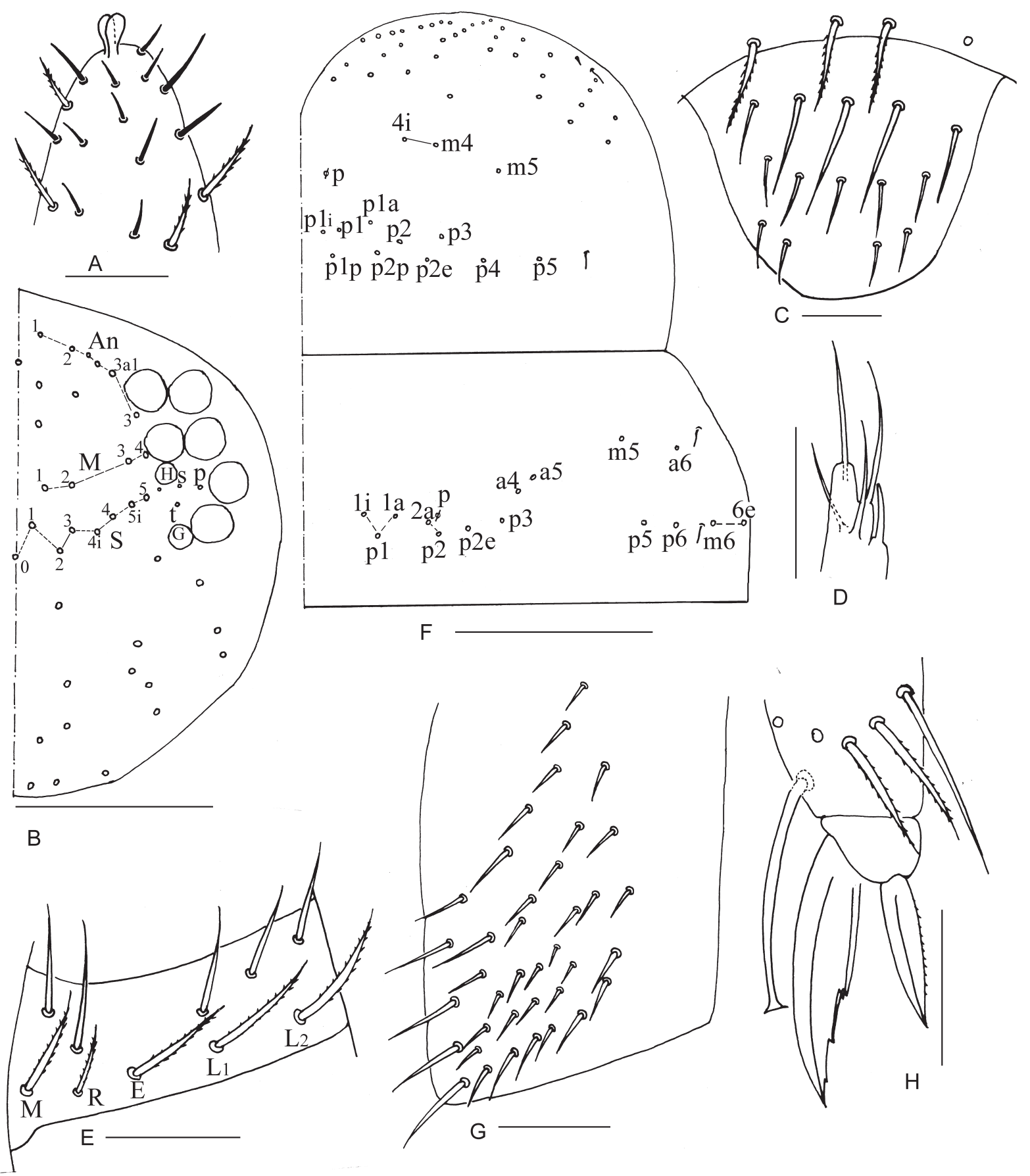

Fig. 2. Willowsia sexachaeta sp. nov. A. Apex of Ant. IV. B. Dorsal chaetotaxy of head. C. Labrum. D. Lateral process of labial palp. E. Labium. F. Chaetotaxy of Th. II-III. G. Trochanteral organ. H. Foot complex. Scale bars: A, C-E, G-H $=20 \mu \mathrm{m}$; B, F $=100 \mu \mathrm{m}$. 

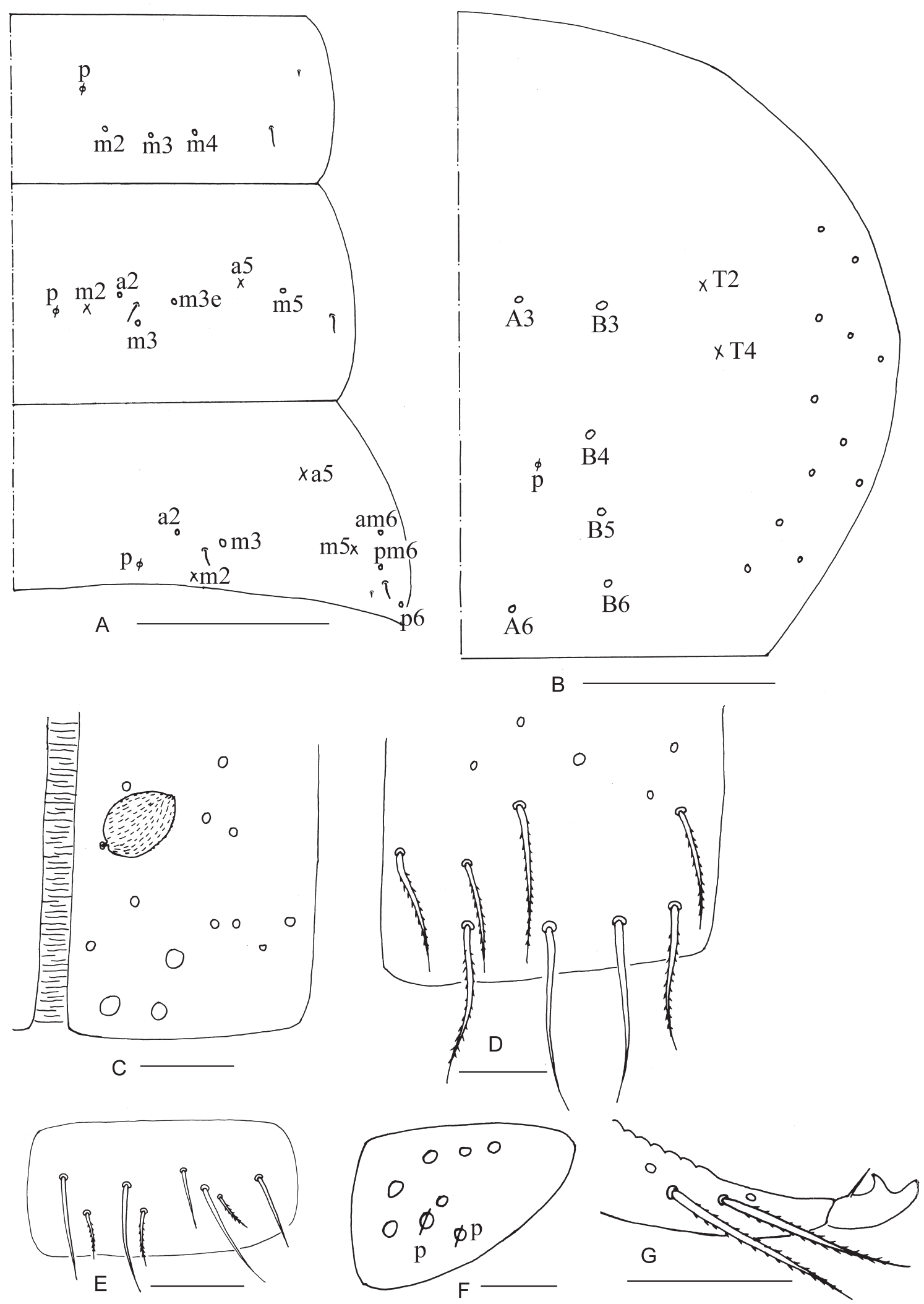

Fig. 3. Willowsia sexachaeta sp. nov. A. Chaetotaxy of Abd. I-III. B. Chaetotaxy of Abd. IV. C. Anterior face of ventral tube. D. Posterior face of ventral tube. E. Lateral flap of ventral tube. F. Manubrial plaque. G. Distal part of dens and mucro. Scale bars: $A-B=100 \mu \mathrm{m} ; C-G=20 \mu \mathrm{m}$. 
Labral chaetae as 4/5, 5, 4, all slender; prelabral chaetae ciliate, other smooth; labral papillae not clearly seen (Fig. 2C). Lateral process of labial palp curved, slightly thicker than normal chaetae, with tip not reaching apex of labial papilla (Fig. 2D). Labial triangular chaetae as $\mathrm{MREL}_{1} \mathrm{~L}_{2}$, all finely ciliate (Fig. 2E).

Thorax. Th. II with 2 (m4, m4i) medio-sublateral, 9-11 posterior mac (p4 sometimes absent, $\mathrm{p} 6$ rarely present), $1 \mathrm{~ms}$ and 2 sens (Fig. 2F). Th. III with 8-9 (p1i sometimes present) median, 6 (m5, a6, p5, p6, m6, m6e) lateral mac and 2 sens, p5 rarely absent (Fig. 2F). Trochanteral organ with 27-38 smooth spiny chaetae (Fig. 2G). Unguis with 4 inner teeth, one pair located about 0.5 from base of inner edge of unguis, distal unpaired two at about 0.7 and 0.9 distance from base respectively. Unguiculus acuminate and outer edge serrate. Tenent hair thick with clavate tip, almost equal to or slightly longer than inner edge of unguis (Fig. 2H).

Abdomen. Abd. IV 3.0-4.5 times as long as Abd. III along dorsal midline. Dorsal mac shown in Fig. 3A-B. Abd. I with $3(\mathrm{~m} 2, \mathrm{~m} 3, \mathrm{~m} 4)$ mac, $1 \mathrm{~ms}$ and 1 sens; sens inner to ms. Abd. II with 3 (a2, m3, m3e) central, 1 (m5) lateral mac and 2 sens. Abd. III with 2 (a2, m3) central, 3 (am6, pm6, p6) lateral mac, $1 \mathrm{~ms}$ and 2 sens (Fig. 3A). Abd. IV with 6 (A3, A6, B3, B4, B5, B6) central and 11-13 lateral mac (Fig. 3B). Ventral tube anteriorly with 11-16 ciliate chaetae (Fig. 3C), posteriorly with 2 apical smooth chaetae and 7-11 ciliate chaetae (Fig. 3D), laterally with 5 smooth and 2-3 ciliate chaetae (Fig. 3E). Manubrial plaque with 6-7 ciliate chaetae and 2 (rarely 3) pseudopores (Fig. 3F). Dens without spines, uncrenulated dens 1.4-1.7 times as mucro in length. Mucro bidentate, two teeth subequal and tip of basal spine reaching subapical tooth (Figs 3G, 4A).

SCALEs. Hyaline, spinulate type, leaf-like and present on Ant. I-II, dorsal side of head and terga, coxa to tibiotarsus, anterior face of ventral tube and ventral side of manubrium (Figs 4B-4C).

\section{Remarks}

The new species is similar to another Chinese species, $W$. baoshanensis Chai \& Ma, 2017, in colour pattern and scale morphology, but the difference between them in chaetotaxy is great. It is also similar to the species W. japonica (Folsom, 1897) in colour pattern on the body and three mac on Abd. I, but scale morphology is of the spinulate type in the former and the short rib type in the latter (Table 1). The differences between the three species are great, as shown in Table 2.

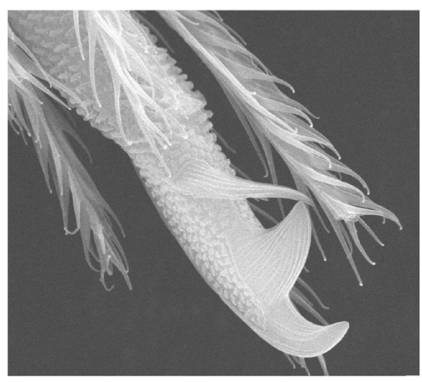

A

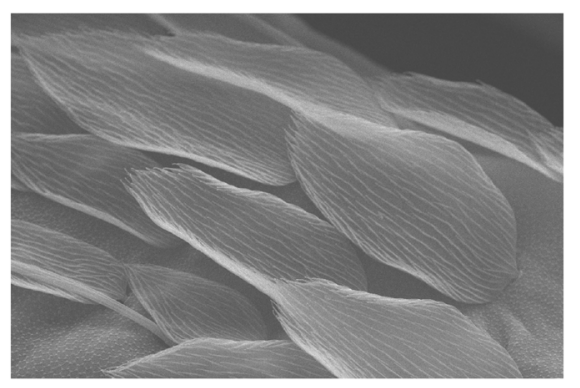

$\mathrm{B}$

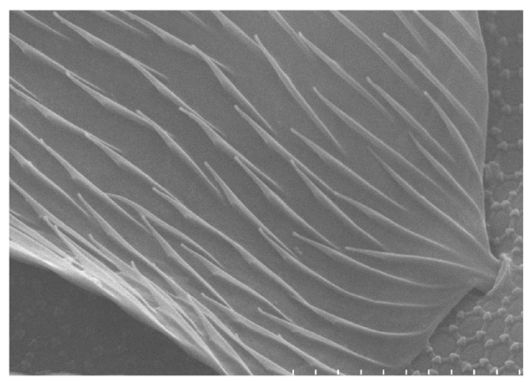

C

Fig. 4. Willowsia sexachaeta sp. nov. A. Distal part of dens and mucro. B-C. Scale(s). Scale bars: $\mathrm{A}=5 \mu \mathrm{m} ; \mathrm{B}=10 \mu \mathrm{m} ; \mathrm{C}=2.5 \mu \mathrm{m}$. 
Willowsia christianseni sp. nov.

urn:1sid:zoobank.org:act:FEBAECA8-BB5C-46FC-95C1-6A194A32330B

Figs 5-7, Tables 1,3

\section{Diagnosis}

Anterior margin of Th. II and lateral margin of Th. II-III with blue pigment, an interrupted blue stripe present along posterior margins of Abd. I and II respectively; Abd. I with 4 mac; Abd. II with 4 central mac; Abd. III with 3 central and 4 lateral mac; Scales spinulate type and present on Ant. I-II, dorsal side of head and terga, coxa to tibiotarsus, anterior face of ventral tube and ventral side of manubrium.

\section{Etymology}

In memory of Kenneth Christiansen, who gave me much help in the taxomony of Collembola.

\section{Material examined}

\section{Holotype}

CHINA: 9 , on slide, collected from outside of Shangdiwan Cave, Louna Village, Dingxiao Town, Xingyi City, Guizhou Province, $25^{\circ} 09^{\prime} 33^{\prime \prime} \mathrm{N}, 105^{\circ} 02^{\prime} 35^{\prime \prime} \mathrm{E}, 1300 \mathrm{~m}$ a.s.l., in rotten stem of maize, 28 Apr. 2014, Shangze Wu leg (Collection number 1144, NTU).

\section{Paratype}

CHINA: 1 , , on slide, same data as holotype.

\section{Description}

Measurements. Body length up to $1.8 \mathrm{~mm}$.

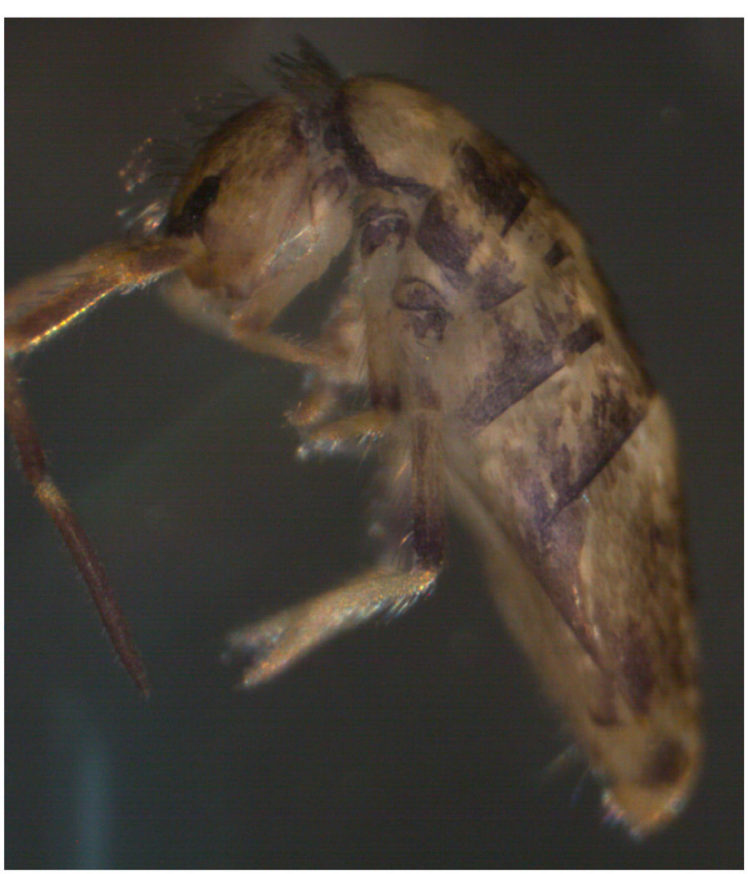

A

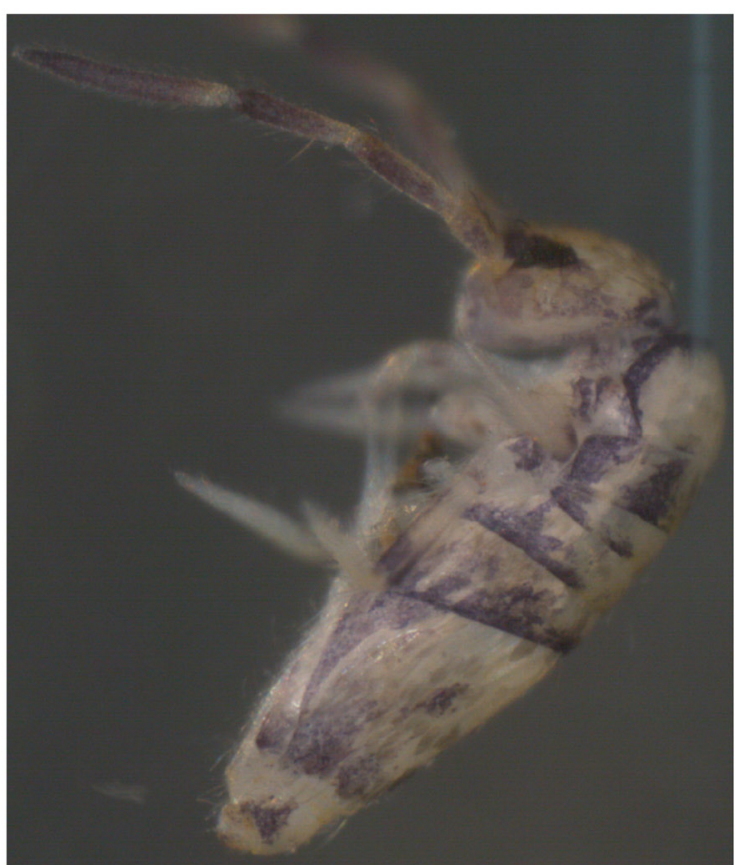

B

Fig. 5. Willowsia christianseni sp. nov. A-B. Habitus. Scale bars: $500 \mu \mathrm{m}$. 
Colour. Ground colour pale yellow. Eyepatches dark blue. Ant. I-IV almost blue pigmented except proximal part of each segment. Anterior margin of Th. II and lateral margin of Th. II-III with blue pigment. An interrupted blue stripe present along posterior margins of Abd. I and II respectively. Blue pigment also present on posterior part of Abd. III- $\mathrm{V}$, distal part of femora of middle and hind legs (Figs 5A-5B).

HEAD. Antenna 0.5-0.6 times as long as body. Antennal segments ratio as I: II: III: IV $=1$ : $1.7-2.2$ : 1.7-2.0: 3.0-3.3. Distal part of Ant. IV with many sensory chaetae, normal ciliate chaetae and a bilobed apical bulb (Fig. 6A). Dorsal cephalic chaetotaxy with 6 antennal (An), 4 median (M) and 8 sutural (S) mac (Fig. 6B). Interocular chaetae as p, s, t, all finely ciliate (Fig. 6B). Eyes $8+8$, G and $\mathrm{H}$ smaller (Fig. 6B). Labral chaetae as 4/5, 5, 4, all slender; prelabral chaetae ciliate, other smooth; distal margin of labrum with 4 papillae, each with a denticle (Fig. 6C). Lateral process of labial palp curved, slightly thicker than normal chaetae, with tip exceeding apex of labial papilla (Fig. 6D). Labial triangular chaetae as in Fig. 6E, MREL $\mathrm{L}_{2}$ all finely ciliate.

Thorax. Dorsal macrochaetae shown as in Fig. 6F. Th. II with 1 (m1) medio-median, 2 (m4, m4i) medio-sublateral and 18 posterior mac, $\mathrm{m} 5$, ms and sens not clearly seen. Th. III with 13 median, 6 (m5, a6, p5, p6, m6, m6e) lateral mac and 2 sens on each side. Trochanteral organ with 21 smooth spiny chaetae (Fig. 6G). Unguis with 4 inner teeth, one pair located 0.5 from base of inner edge of unguis, distal unpaired two at 0.8 and 0.9 distance from base respectively. Unguiculus acuminate and outer edge serrate. Tenent hair thick with clavate tip, slightly longer than length of inner edge of unguis (Fig. $6 \mathrm{H}$ ).

Abdomen. Abd. IV 4.0 times as long as Abd. III along dorsal midline. Dorsal mac shown in Figs 7A-7B. Abd. I with 4 (m2, m3, m4, a5) mac, 1 ms and 1 sens; sens inner to ms. Abd. II with 4 (a2, m3, m3e, m3ep) central, 1 (m5) lateral mac and 2 sens. Abd. III with 3 (a2, a3, m3) central, 4 (am6, pm6, p6, m7) lateral mac, $1 \mathrm{~ms}$ and 2 sens (Fig. 7A). Abd. IV with 10-12 central (A3, A4, A6, B3, B4, B5, B6 always present) and about 14 lateral mac as in Fig. 7B. Ventral tube anteriorly with about 15 ciliate chaetae (Fig. 7C), posteriorly and laterally not clearly seen. Dens without spines, uncrenulated dens about $2.5 \times$ as long as mucro in. Mucro bidentate, two teeth subequal and tip of basal spine reaching subapical tooth (Fig. 7D).

SCALEs. Hyaline, spinulate type, leaf-like and present on Ant. I-II, dorsal side of head and terga, coxa to tibiotarsus, anterior face of ventral tube and ventral side of manubrium (Fig. 7E).

\section{Remarks}

The new species is similar to the species W. nigromaculata (Lubbock, 1873) in colour pattern, but scale morphology is of the spinulate type in the former and the long basal rib type in the latter. It is also similar to another species from Guizhou Province, W. pseudoshi Ma, 2016, in chaetotaxy of Th. III and Abd. I-II, but the colour pattern and chaetotaxy of Abd. III-IV are not the same. The differences between the three species are great, as shown in Table 3. 


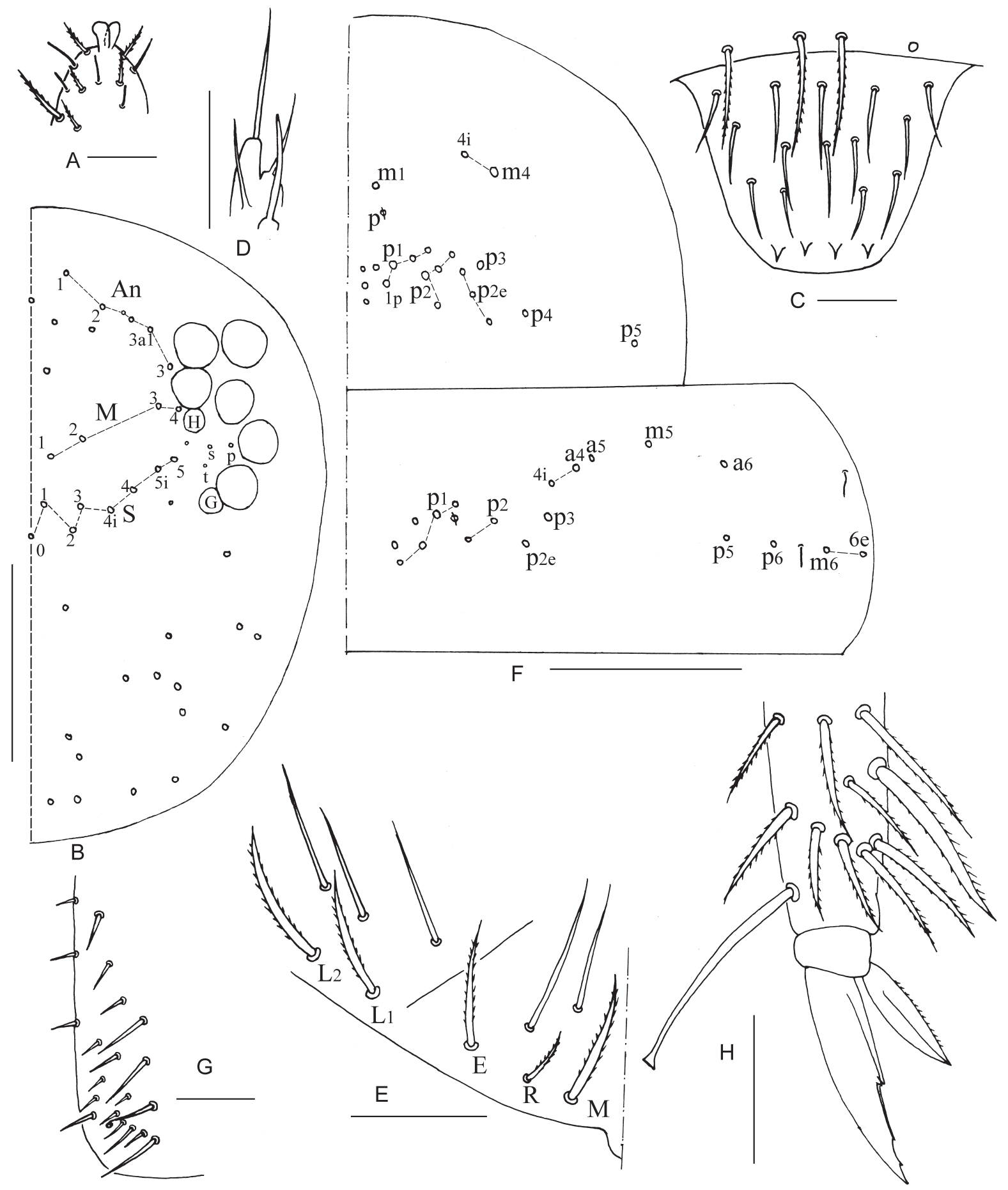

Fig. 6. Willowsia christianseni sp. nov. A. Apex of Ant. IV. B. Dorsal chaetotaxy of head. C. Labrum. D. Lateral process of labial palp. E. Labium. F. Chaetotaxy of Th. II-III. G. Trochanteral organ. H. Foot complex. Scale bars: A, C-E, G-H $=20 \mu \mathrm{m} ; \mathrm{B}, \mathrm{F}=100 \mu \mathrm{m}$. 


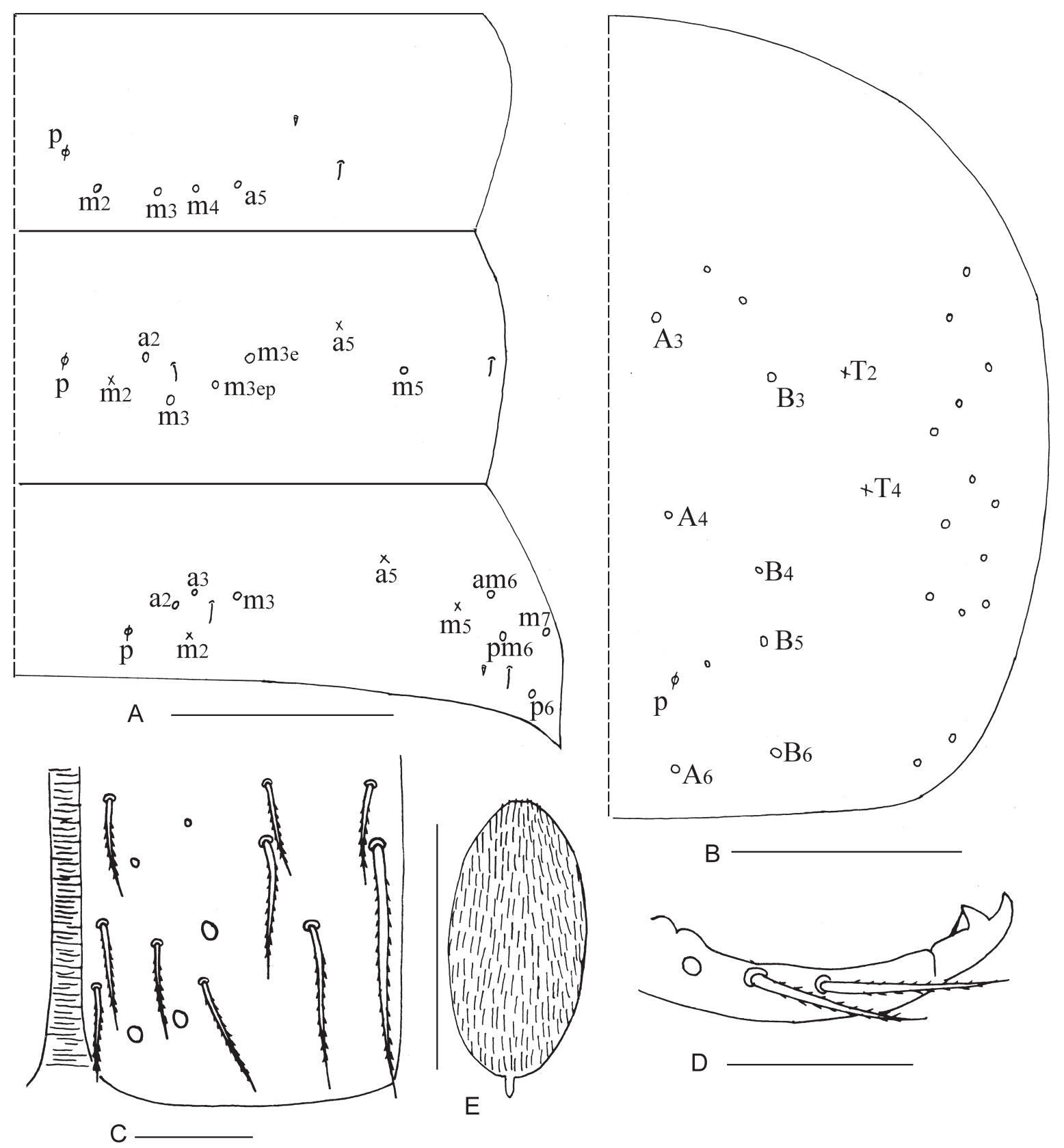

Fig. 7. Willowsia christianseni sp. nov. A. Chaetotaxy of Abd. I-III. B. Chaetotaxy of Abd. IV. C. Anterior face of ventral tube. D. Distal part of dens and mucro. E. Scale. Scale bars: A-B = $100 \mu \mathrm{m}$; $\mathrm{C}-\mathrm{E}=20 \mu \mathrm{m}$. 
Table 3. Comparison of Willowsia christianseni sp. nov., W. nigromaculata (Lubbock, 1873) and W. pseudoshi Ma, 2016.

\begin{tabular}{|c|c|c|c|}
\hline Characters & W. christianseni sp. nov. & W. nigromaculata & W.pseudoshi \\
\hline Colour of antennae & blue & $\begin{array}{l}\text { yellow with blue } \\
\text { pigment }\end{array}$ & yellow \\
\hline Colour of head & yellow with blue patches & $\begin{array}{l}\text { yellow with blue } \\
\text { patches }\end{array}$ & $\begin{array}{l}\text { almost blue } \\
\text { wholly }\end{array}$ \\
\hline Scale type & spinulate & long basal rib & spinulate \\
\hline Median mac on dorsal head & 4 & unknown & 5 \\
\hline Posterior mac on Th. II & 18 & 7 & $17-19$ \\
\hline Central mac on Th. III & 13 & 4 & $15-16$ \\
\hline Mac on Abd. I & 4 & 3 & 4 \\
\hline M3ep on Abd. II & present & absent & present \\
\hline Central mac on Abd. III & 3 & 3 & 2 \\
\hline Lateral mac on Abd. III & 4 & 3 & 5 \\
\hline Central mac on Abd. IV & $10-12$ & 7 & $16-19$ \\
\hline
\end{tabular}

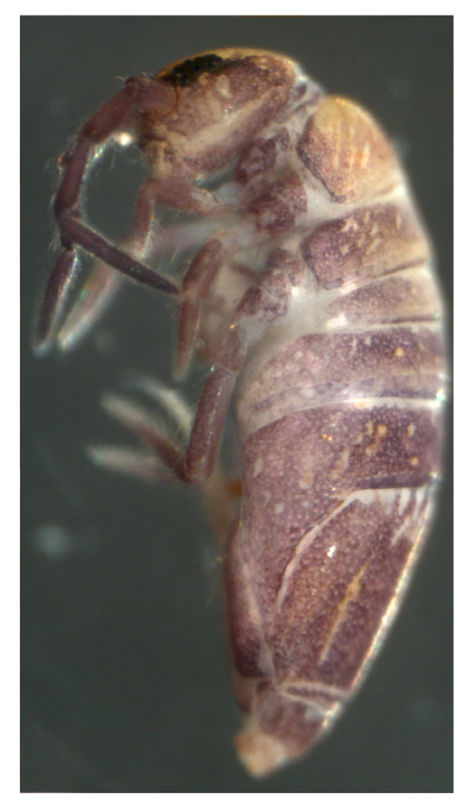

A

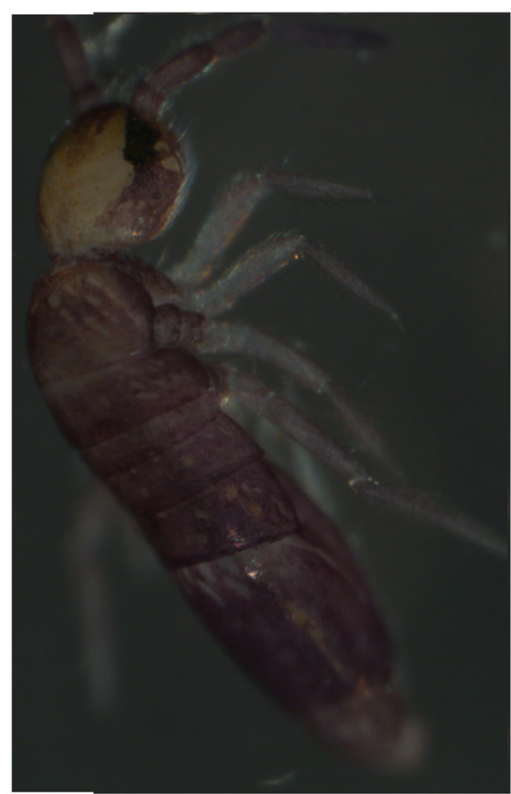

$\mathrm{B}$

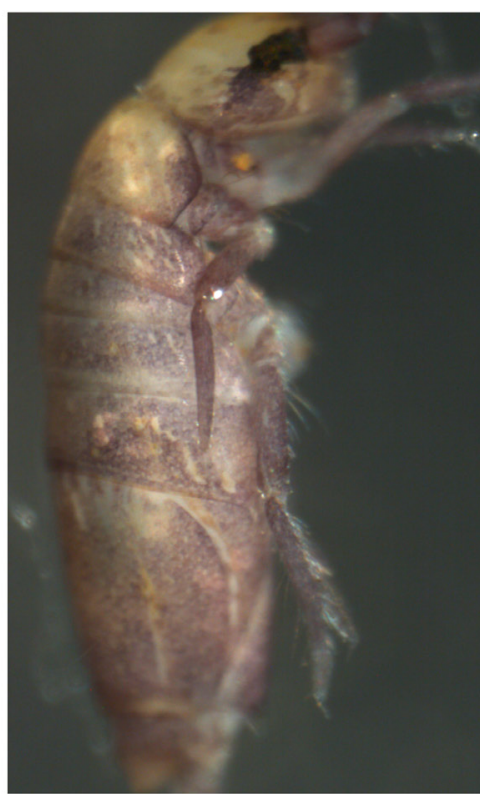

$\mathrm{C}^{\prime}$

Fig. 8. Willowsia tanae sp. nov. A-C. Habitus. Scale bars: $500 \mu \mathrm{m}$. 


\author{
Willowsia tanae sp. nov. \\ urn:1sid:zoobank.org:act:070A4536-56EA-4325-A733-2901AF6512A4 \\ Figs 8-10, Tables 1, 4
}

\title{
Diagnosis
}

Antennae and legs almost wholly blue pigmented and blue pigment present on most part of dorsum from Th. III to Abd. V; Abd. I with 3 mac; Abd. II with 3 central mac; Abd. III with 3 central and 3 lateral mac; Scales spinulate type and present on Ant. I-II, dorsal side of head and terga, coxa to tibiotarsus, anterior face of ventral tube and ventral side of manubrium.

\section{Etymology}

Named after Ms Hongdan Tan, who collected the specimens.

\section{Material examined}

\section{Holotype}

CHINA: + , on slide, Shangkanzhe Village, Laochang Town, Pan County, Guizhou Province, 2539'34" N, 104²' 27" E, 1366 m a.s.1., in litter, 22 Jan. 2016, Hongdan Tan leg. (Collection number 1166, NTU).

\section{Paratypes}

CHINA: 6 우, on slide, same data as holotype.

\section{Description}

Measurement. Body length up to $1.5 \mathrm{~mm}$.

CoLour. Ground colour pale yellow. Eyepatches dark blue. Antennae and legs almost wholly blue pigmented. Lateral side of Th. II and head behind eyepatches with blue pigment. Blue pigment present on most part of dorsum from Th. III to Abd. V (Figs 8A-8C).

HEAD. Antenna $0.3-0.4 \times$ as long as body. Antennal segments ratio as I: II: III: IV = 1: $1.4-1.7: 1.4-1.7$ : 2.1-2.9. Distal part of Ant. IV with many sensory chaetae, normal ciliate chaetae and a bilobed apical bulb (Fig. 9A). Dorsal cephalic chaetotaxy with 7-8 antennal (An), 4 median (M) and 9 sutural (S) mac (Fig. 9B). Interocular chaetae as p, s, t, all finely ciliate (Fig. 9B). Eyes 8+8, G and H smaller (Fig. 9B). Labral chaetae as 4/5, 5, 4, all slender; prelabral chaetae ciliate, other smooth, labral papillae not clearly seen (Fig. 9C). Lateral process of labial palp almost as thick as normal chaetae, with tip not reaching apex of labial papilla (Fig. 9D). Subapical chaeta of maxillary outer lobe equal to apical one, three smooth hairs on sublobal plate (Fig. 9E). Labial triangular chaetae as in Fig. 9F, MREL $\mathrm{L}_{2}$ all finely ciliate.

Thorax. Dorsal macrochaetae shown as in Fig. 9G. Th. II with 2 (m1, m2) medio-median, 3 (m4, m4i, $\mathrm{m} 4 \mathrm{p})$ medio-sublateral, 13 posterior mac, $1 \mathrm{~ms}$ and 2 sens. Th. III with $9(10, \mathrm{p} 1 \mathrm{p}$ rarely present $)$ median, 6-7 (m5, a6, p5, p6, m6, m6e, m5i sometimes absent) lateral mac and 2 sens. Trochanteral organ with 25-26 smooth spiny chaetae (Fig. 9H). Unguis with 4 inner teeth, one pair located $0.5-0.6$ from base of inner edge of unguis, distal unpaired two respectively at 0.7 and 0.9 distance from base. Unguiculus acuminate and outer edge serrate. Tenent hair thick with clavate tip, slightly longer than length of inner edge of unguis (Fig. 9I).

Abdomen. Abd. IV 2.5-3.2 $\times$ as long as Abd. III along dorsal midline. Dorsal mac shown in Figs 10A-10B. Abd. I with 3 (m2, m3, m4) mac, 1 ms and 1 sens; sens inner to ms. Abd. II with 3 (a2, $\mathrm{m} 3, \mathrm{~m} 3 \mathrm{e}$ ) central, 1 (m5) lateral mac and 2 sens. Abd. III with 1 (m3) central, 3 (am6, pm6, p6) lateral mac, 1 ms and 2 sens (Fig. 10A). Abd. IV with 7 central (A3, A4, A6, B3, B4, B5, B6) and 11-13 lateral 

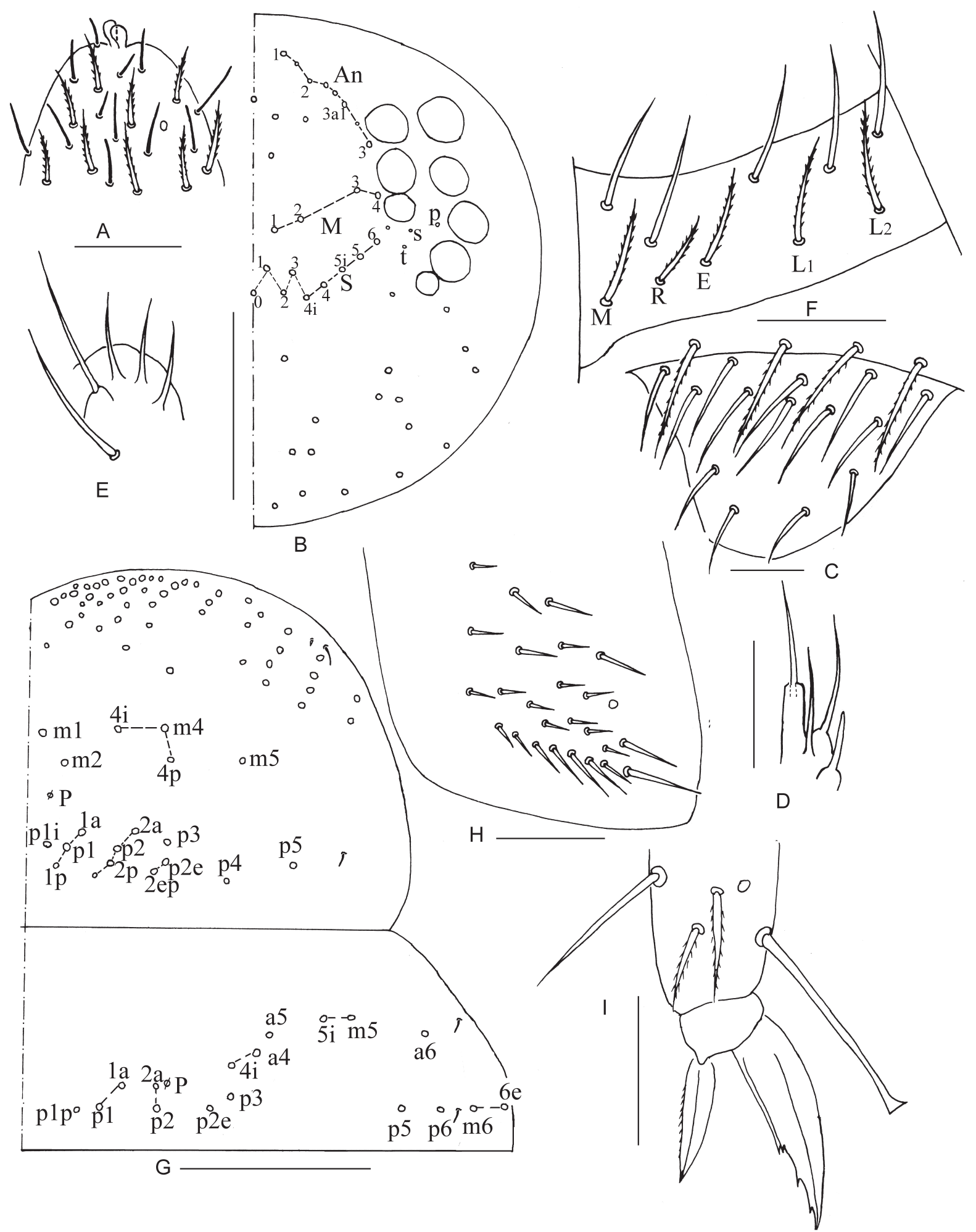

Fig. 9. Willowsia tanae sp. nov. A. Apex of Ant. IV. B. Dorsal chaetotaxy of head. C. Labrum. D. Lateral process of labial palp. E. Maxillary outer lobe. F. Labium. G. Chaetotaxy of Th. II-III. H. Trochanteral organ. I. Foot complex. Scale bars: A, C-F, H-I $=20 \mu \mathrm{m} ; \mathrm{B}, \mathrm{G}=100 \mu \mathrm{m}$. 

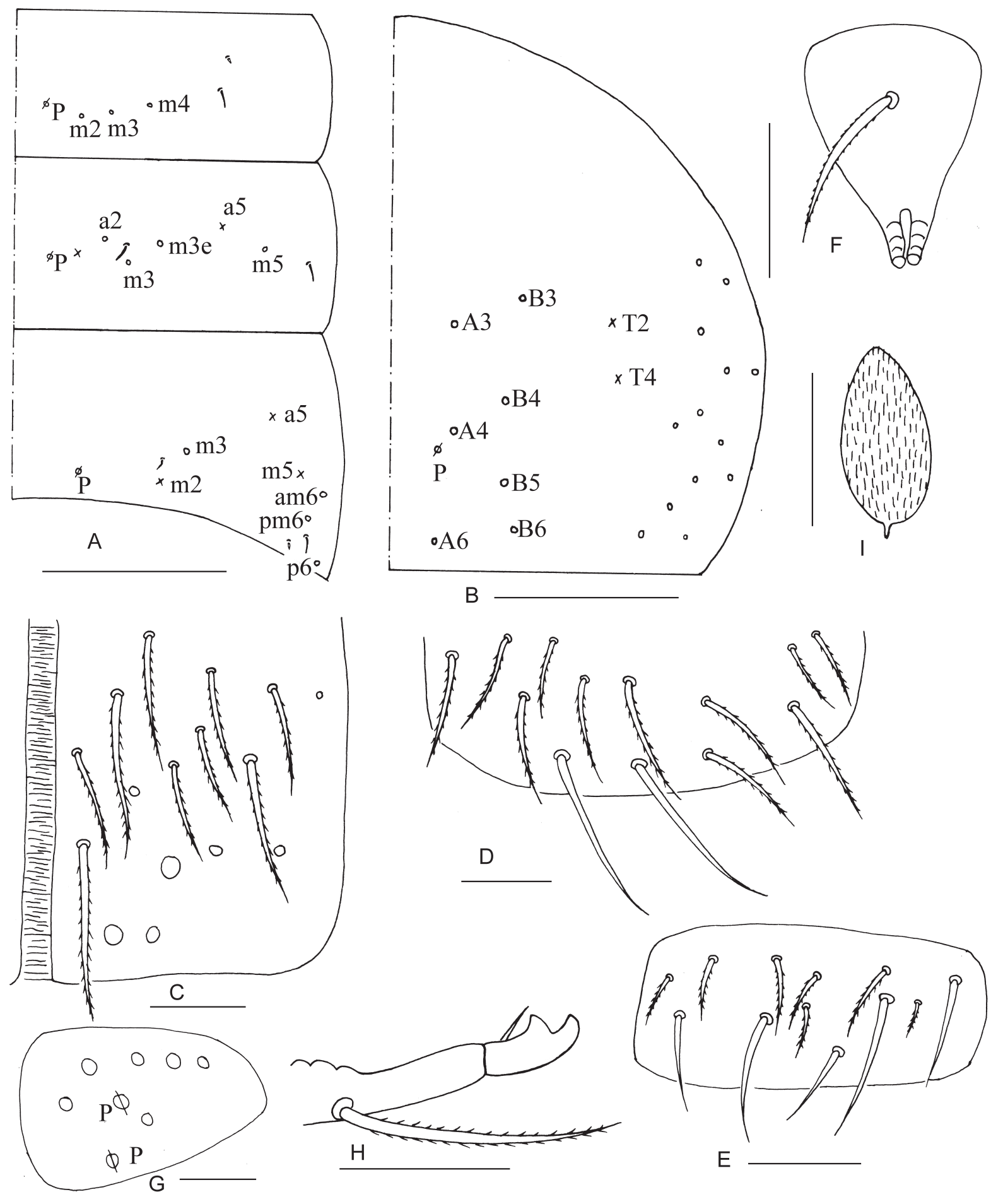

Fig. 10. Willowsia tanae sp. nov. A. Chaetotaxy of Abd. I-III. B. Chaetotaxy of Abd. IV. C. Anterior face of ventral tube. D. Posterior face of ventral tube apically. E. Lateral flap of ventral tube. F. Tenaculun. G. Manubrial plaque. H. Distal part of dens and mucro. I. Scale. Scale bars: A-B $=100 \mu \mathrm{m} ; \mathrm{C}-\mathrm{I}=20 \mu \mathrm{m}$. 
Table 4. Comparison of Willowsia. tanae sp. nov., W. variabilis Ma, 2016 and W. sexachaeta sp. nov

\begin{tabular}{lccc}
\hline Characters & W. tanae sp. nov. & W. variabilis & W. sexachaeta sp. nov \\
\hline Blue pigment on body & much & much & little \\
Medio-median mac on Th. II & 2 & 2 & 0 \\
Posterior mac on Th. II & 13 & $23-25$ & $9-11$ \\
Central mac on Th. III & $9-10$ & $17-18$ & $7-8$ \\
Mac on Abd. I & 3 & $7-8$ & 3 \\
Central mac on Abd. II & 3 & 5 & 3 \\
Central mac on Abd. III & 1 & 2 & $1-2$ \\
Central mac on Abd. IV & 7 & $10-13$ & 6 \\
\hline
\end{tabular}

mac as in Fig. 10B. Ventral tube anteriorly with 16-18 ciliate chaetae (Fig. 10C), posteriorly with 24 ciliate chaetae and 2 smooth chaetae (Fig. 10D), laterally with 6-7 ciliate chaetae and 5 smooth chaetae (Fig. 10E). Tenaculum with a striate chaeta (Fig. 10F). Manubrial plaque with 5-6 ciliate chaetae and 2 pseudopores (Fig. 10G). Dens without spines, uncrenulated dens 1.1-1.2 times as mucro in length. Mucro bidentate, two teeth subequal and tip of basal spine reaching subapical tooth (Fig. 10H).

SCALEs. Hyaline, spinulate type, leaf-like and present on Ant. I-II, dorsal side of head and terga, coxa to tibiotarsus, anterior face of ventral tube and ventral side of manubrium (Fig. 10I).

\section{Remarks}

The new species is most similar to the Chinese species $W$. variabilis Ma, 2016 in colour pattern and scale type, but Abd. I-IV with 3, 3, 1 and 7 central mac in the former respectively, but 7-8, 5, 2, 10-13 central mac in the latter. It is also similar to the species $W$. sexachaeta sp. nov. in chaetotaxy, but their colour patterns are different. The differences between the three species are shown in Table 4.

\section{Discussion}

Scale morphology is a major character in defining species of the genus Willowsia and it was divided into four types (Zhang et al. 2011): spinulate type, short rib type, long basal rib type and uninterrupted type. Among 20 Chinese species of the genus, nine species have scales of the spinulate type and they were described or reported only from the Southwest of China, including Guangxi, Guizhou, Tibet and Yunnan provinces. Ten species with the long basal rib type and one species with the short rib type were from other provinces except the Southwest of China. No species with the uninterrupted scale type have been found in China.

\section{Acknowledgements}

Thanks are given to Feng Zhang (Nanjing Agricultural University) and anonymous referees who provided kind advice on this manuscript.

\section{References}

Chai R. \& Ma Y.T. 2017. Two new species of Willowsia (Collembola: Entomobryidae) from Yunnan Province, China. European Journal of Taxonomy 311: 1-12. https://doi.org/10.5852/ejt.2017.311

Folsom J.W. 1897. Japanese Collembola. Bulletin of the Essex Institute 29: 51-57. https://doi.org/10.5962/bhl.part.14789 
Gisin H. 1967. Espèces nouvelles et lignées évolutives de Pseudosinella endogés (Collembola). Memórias e Estudos do Museu Zoológico da Universidade de Coimbra 301: 5-25.

Jordana R. \& Baquero E. 2005. A proposal of characters for taxonomic identification of Entomobrya species (Collembola, Entomobryomorpha), with description of a new species. Abhandlungen und Berichte des Naturkundemuseums Goerlitz 76 (2): 117-134.

Lubbock J. 1873. Monograph of the Collembola and Thysanura. Ray Society, London. https://doi.org/10.5962/bhl.title.11583

Ma Y.T. 2016. Two new species of Willowsia (Collembola, Entomobryidae) from Guizhou Province, China. Zootaxa 4158 (1): 137-145. https://doi.org/10.11646/zootaxa.4158.1.9

Mari-Mutt J.A. 1986. Puerto Rican species of Lepidocyrtus and Pseudosinella (Collembola: Entomobryidae). Caribbean Journal of Science 22 (1-2): 1-48.

Szeptycki A. 1979. Morpho-systematic studies on Collembola. IV. Chaetotaxy of the Entomobryidae and its phylogenetical significance. Polska Akademia Nauk, Kraków.

Zhang F., Chen J.X. \& Deharveng L. 2011. New insight into the systematics of the Willowsia complex (Collembola: Entomobryidae). Annales de la Société entomologique de France 47 (1-2): 1-20. https://doi.org/10.1080/00379271.2011.10697692

Manuscript received: 4 June 2018

Manuscript accepted: 20 August 2018

Published on: 26 October 2018

Topic editor: Gavin Broad

Desk editor: Jeroen Venderickx

Printed versions of all papers are also deposited in the libraries of the institutes that are members of the EJT consortium: Muséum national d'Histoire naturelle, Paris, France; Botanic Garden Meise, Belgium; Royal Museum for Central Africa, Tervuren, Belgium; Natural History Museum, London, United Kingdom; Royal Belgian Institute of Natural Sciences, Brussels, Belgium; Natural History Museum of Denmark, Copenhagen, Denmark; Naturalis Biodiversity Center, Leiden, the Netherlands; Museo Nacional de Ciencias Naturales-CSIC, Madrid, Spain; Real Jardín Botánico de Madrid CSIC, Spain. 